\title{
Newborns in England are screened for four extra genetic conditions
}

In this News story (BMJ 2015;350:h10, doi:10.1136/bmj.h10), we inadvertently said that the "heel prick" blood test on newborn babies is presently used to test for hyperthyroidism. That was

incorrect. We should have said that it was used to test for

hypothyroidism. We apologise for this mistake. 